\title{
Multiscale Modelling of Graphene from Nano to Micron Scales
}

\author{
Tapio Ala-Nissila \\ Department of Applied Physics and COMP CoE \\ Aalto University School of Science \\ P.O. Box 11000 \\ FIN-0076 Aalto, Espoo \\ Finland
}

Over the last few years novel two-dimensional materials and nanoscopically thin heteroepitaxial overlayers have attracted intense attention due to their unusual properties and important technological applications. Many physical properties of these systems such as thermal conductivity and electrical transport, are intimately coupled to the large scale mechanical and structural properties of the materials. However, modeling such properties is a formidable challenge due to a wide span of length and time scales involved. In this talk, I will review recent significant progress in structural multi-scale modeling of two-dimensional materials and thin heteroepitaxial overlayers [1], and graphene in particular, based on the Phase Field Crystal (PFC) model. The PFC model allows one to reach diffusive time scales for structural relaxation of the materials at the atomic scale, which facilitates quantitative characterisation of domain walls, dislocations, grain boundaries, and straindriven self-organisation up to micron length scales [1]. This allows one to study thermal conduction and electrical transport in realistic multi-grain systems.

\section{References}

[1] K. R. Elder et al, Phys. Rev. Lett., vol. 108, 226102, 2012; Phys. Rev. B, vol. 88, 075423, 2013. 